\title{
Technology development for the Constellation-X hard-X-ray telescope
}

Fiona A. Harrison, Walter R. Cook, Finn Erland Christensen, Oberto Citterio, William W. Craig, et al.

Fiona A. Harrison, Walter R. Cook, Finn Erland Christensen, Oberto Citterio, William W. Craig, Neil A. Gehrels, Paul Gorenstein, Jonathan E. Grindlay, Charles J. Hailey, Richard A. Kroeger, Hideyo Kunieda, Giovanni Pareschi, Ann M. Parsons, Robert Petre, Suzanne E. Romaine, Brian D. Ramsey, Jack Tueller, Melville P. Ulmer, Martin C. Weisskopf, David L. Windt, "Technology development for the Constellation-X hard-x-ray telescope," Proc. SPIE 3765, EUV, X-Ray, and Gamma-Ray Instrumentation for Astronomy X, (22 October 1999); doi: $10.1117 / 12.366492$ Instrumentation, 1999, Denver, CO, United States 


\title{
Technology development for the Constellation-X hard X-ray telescope
}

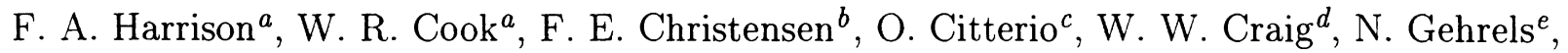 \\ P. Gorenstein ${ }^{f}$, J. E. Grindlay ${ }^{g}$, C. J. Hailey ${ }^{d}$, R. A. Kroeger ${ }^{h}$, H. Kuneida ${ }^{i}$, \\ G. Pareschi ${ }^{c}$, A. M. Parsons ${ }^{e}$, R. Petre ${ }^{e}$, S. E. Romaine ${ }^{f}$, B. D. Ramsey ${ }^{j}$, \\ J. Tueller ${ }^{e}$, M. Ulmer ${ }^{l}$, M. C. Weisskopf ${ }^{j}$, D. W. Windt ${ }^{k}$ \\ ${ }^{a}$ Caltech 220-47, Pasadena, CA 91125 \\ ${ }^{b}$ Danish Space Research Instititute, Juliane Maries Vej 30 \\ Copenhagen Dk-2100, Denmark \\ ${ }^{c}$ Osservatorio Astronomico di Brera-Milano, Merate, It \\ ${ }^{d}$ Columbia Astrophysics Laboratory, Columbia University, NY \\ ${ }^{e}$ Goddard Space Flight Center, Greenbelt, MD

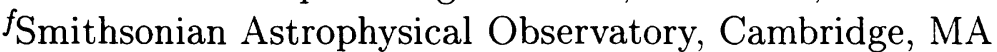 \\ ${ }^{g}$ Harvard College Observatory, Cambridge, MA \\ ${ }^{h}$ Naval Research Laboratories, Washington, DC \\ ${ }^{i}$ Nagoya University, Nagoya, Japan \\ ${ }^{j}$ Marshall Space Flight Center, Huntsville, AL \\ ${ }^{k}$ Lucent Technologies, Murray Hill, NJ \\ ${ }^{l}$ Northwestern University, Evanston, IL
}

\begin{abstract}
In addition to high resolving power in the traditional $\mathrm{X}$-ray band, the Constellation-X scientific goals require broad bandpass, with response extending to $\mathrm{E} \gtrsim 40 \mathrm{keV}$. To achieve this objective, Constellation- $\mathrm{X}$ will incorporate a hard X-ray telescope (HXT) based on depth graded multilayer-coated grazing incidence optics and position-sensitive solid state detectors. This paper describes the HXT performance requirements, provides an overview of the HXT optics and detector technology development efforts, and presents example designs.
\end{abstract}

Keywords: Hard X-ray Telescopes, X-ray Optics, Multilayers, Hard X-ray Detectors, X-ray Missions

\section{INTRODUCTION}

A number of the key Constellation-X scientific objectives require extending the instrument sensitivity into the hard $\mathrm{X}$ ray band, up to energies $>40 \mathrm{keV}$. For AGN in particular, hard X-ray measurements of the continuum are required in order to fully interpret Iron-line features, and therefore constrain emission mechanisms. In Seyfert galaxies, measurements of the continuum shape at high energies can be used to determine the magnitude of the Compton reflection component and thereby provide constraints on the viewing angle and source geometry. Other examples of scientific investigations where sensitivity in the hard X-ray is important include studies of stellar flares and shock emission from young supernova remnants.

To-date, the high-sensitivity made possible by grazing-incidence X-ray optics has been limited to energies below $\sim 10 \mathrm{keV}$. To extend focusing into the hard X-ray band, numerous groups are developing depth-graded multilayercoated optics and position-sensitive high atomic number solid state detectors. The Hard X-ray Telescope (HXT) instrument, to be co-aligned with the Spectroscopy X-ray Telescope (SXT), plans to incorporate these technologies in order to provide a broad bandpass for the observatory.

In this paper, we describe the HXT performance requirements, provide an overview of the optics and detector technology development efforts - including highlights of recent results - and finally we present example designs for the experiment.

Further author information: (Send correspondence to F.A.H.)

F.A.H.: E-mail: fiona@srl.caltech.edu 


\section{PERFORMANCE REQUIREMENTS}

Table 1 summarizes the HXT performance requirements. The primary performance goal is to match the spectroscopic sensitivity of the SXT for high-energy continuum observations of point sources. This sets the minimum effective area of $\geq 1500 \mathrm{~cm}^{2}$ in the $6-40 \mathrm{keV}$ band, as well as the requirement that typical observations $\left(\mathrm{T}_{o b s} \sim 2 \times 10^{4} \mathrm{~s}\right)$ be signal-limited. The desire to map non-thermal emission in extended sources sets the additional requirement that the field of view be $8^{\prime}$ or greater up to $40 \mathrm{keV}$, and that the HPD be $1^{\prime}$ or better. In addition to the baseline requirements, the Facility Science Team (FST) has established desirable performance enhancements, also listed in Table 1.

\begin{tabular}{|c|c|}
\hline \multicolumn{2}{|c|}{ Baseline HXT Requirements } \\
\hline Effective Area & $\geq 1500 \mathrm{~cm}^{2}, 6-40 \mathrm{keV}$ \\
\hline Signal to Background Ratio & $>1$ for $\mathrm{T}_{o b s} \lesssim 2 \times 10^{4} \mathrm{~s}$ \\
\hline Field of View & $8^{\prime}(6-40 \mathrm{keV})$ \\
\hline HPD & $\lesssim 1^{\prime}$ \\
\hline$\Delta \mathrm{E} / \mathrm{E}$ & $\leq 20 \%$ at $6-30 \mathrm{keV}$ \\
\hline \multicolumn{2}{|c|}{ Desirable Performance Enhancements } \\
\hline Signal to Noise & $>1$ for $\mathrm{T}_{o b s} \lesssim 10^{5} \mathrm{~s}$ \\
\hline Effective Area & $\geq 800 \mathrm{~cm}^{2} 40-80 \mathrm{keV}$ \\
\hline Bandpass & extend to $1 \mathrm{keV}$ \\
\hline HPD & $\lesssim 30^{\prime \prime}$ \\
\hline$\Delta \mathrm{E} / \mathrm{E}$ & $\leq 5 \%$ at $40 \mathrm{keV}$ \\
\hline \multicolumn{2}{|c|}{ Mechanical Envelope } \\
\hline Total mass/satellite (excluding structure) & $\leq 250 \mathrm{~kg}$ \\
\hline Geometric Aperture & $<0.75 \mathrm{~m}^{2}$ \\
\hline Focal Length & $10 \mathrm{~m}$ \\
\hline
\end{tabular}

Table 1. Performance requirements for the HXT. Also shown are desirable enhancements, in order of priority, which set additional technical goals for the instrument, and the mechanical envelope.

The mechanical envelope for the current four-satellite configuration requires a total experiment mass, excluding support structure and optical bench, of $\leq 250 \mathrm{~kg}$, with a focal length of $10 \mathrm{~m}$.

\section{OVERVIEW OF TECHNICAL APPROACH}

The technical challenge associated with extending traditional grazing incidence optics into the hard X-ray band (E $>10 \mathrm{keV}$ ) is the decrease with energy in graze angle for which significant reflectivity can be achieved. For a Wolter or conical approximation mirror geometry, the graze angle, $\gamma$, on a given mirror shell is related to the focal ratio by $\gamma=1 / 4 \times(r / f)$, where $r$ is the shell radius and $f$ is the focal length. If one maintains this geometry, two approaches to extending mirror reflectivity to high energy are possible:

1. utilize small focal ratios $(r / f)$. By using multiple telescope modules of small radius in a highly-nested geometry, low graze-angle hard X-ray telescopes can be designed using standard metal reflective coatings.

2. increase, for a given $r / f$, the graze angle for which significant reflectivity can be achieved. Coating the reflective surfaces with depth-graded multilayer structures, which operate on the principal of Bragg reflection, can substantially increase the maximum graze angle for which significant reflectivity is achieved over a relatively broad energy range.

Due to the larger field of view (resulting from the larger average mirror graze angle) and smaller number of telescope modules, the HXT will employ depth-graded multilayer optics in a Wolter-I or conical-approximation geometry. As described below, this can provide the desired FOV, energy coverage, and effective area with concentration factors (ratio of collecting area to effective detector area) exceeding 5000. In the following section, we describe the specific technologies currently being developed for the HXT. 


\section{TECHNOLOGY DEVELOPMENT}

Several approaches are being pursued by numerous groups to develop hard X-ray optics and focal plane detectors for balloon-borne telescopes and for Constellation-X. A detailed description of all of these is not within the scope of this paper, however in this section we provide an overview of these efforts, with references to more detailed papers.

\subsection{Optics}

Two possible approaches are being pursued for the Con-X mirror substrates: segmented shells in a conical approximation geometry, as used on $A S C A$ and $A s t r o-E^{1}$; and integral replica Wolter-I shells, as employed on $S A X$ and $X M M{ }^{2}$ The former have the advantage that low-mass substrates have been developed that are capable of achieving the requisite angular resolution within the mass envelope, while the latter are attractive due to the superior angular resolution already demonstrated for the $X M M$ optics, although a mass reduction is required.

\subsubsection{Segmented optics}

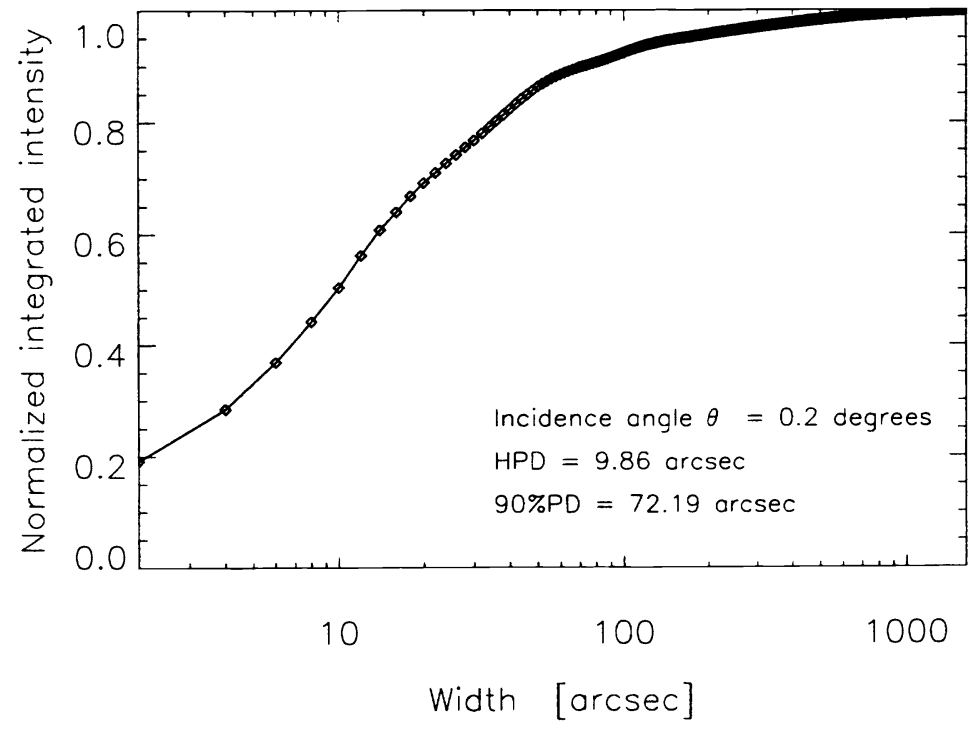

Figure 1. Total encircled energy distribution for $8 \mathrm{keV} \mathrm{X-ray} \mathrm{measurements} \mathrm{on} \mathrm{a} \mathrm{free-standing} \mathrm{thermally-formed}$ glass shell. The measured HPD is $9.86^{\prime \prime}$ for this shell (one bounce). Typical values range from $10-30^{\prime \prime}$.

For segmented hard X-ray optics, the most attractive substrate is thermally formed glass microsheet, as it provides a smooth surface appropriate for multilayer deposition. ${ }^{3}$ In contrast to Aluminum foil optics, it does not require an additional epoxy replication step to remove intermediate-lengthscale ripple. Developed for the CIT/Columbia/DSRI $H E F T$ balloon program, the individual shells have demonstrated figure adequate to achieve the $1^{\prime} \mathrm{HPD}_{\mathrm{goal}}{ }^{4}$ (see Figure 1), and good reflectance for graded multilayers deposited directly on the glass surface. ${ }^{5}$ Glass segments coated with complex depth-graded multilayers do not show any figure degradation due to film stress, and adequate coating uniformity can be achieved using standard planar magnetron systems. ${ }^{5}$ Recently, X-ray testing of an opticalmechanical prototype (shown in Figure 2) with 10 coated and 10 uncoated shells mounted in a flight-representative configuration demonstrated that mirror resolution of $1^{\prime} \mathrm{HPD}$ is possible. Refinement of the mounting procedure is necessary to achieve this performance over the full optic, and will likely improve the HPD beyond the baseline specification.

\subsubsection{Replica optics}

Integral electroformed shells are an attractive option for the HXT due to the superior angular resolution $\left(10^{\prime \prime}\right)$ already demonstrated for the $X M M$ flight optics. Integral shells have the intrinsic advantage of fewer pieces to mount and align. The outstanding technical issues are 1) the substrate mass, which in current thicknesses exceeds the HXT mass limit by a factor $\sim 3$, and 2) coating the interiors of the closed shells. Promising approaches are being pursued to overcome both of these obstacles. New nickel alloys developed for the Con-X SXT ${ }^{6}$ may allow shells as thin as $100 \mu \mathrm{m}$ 


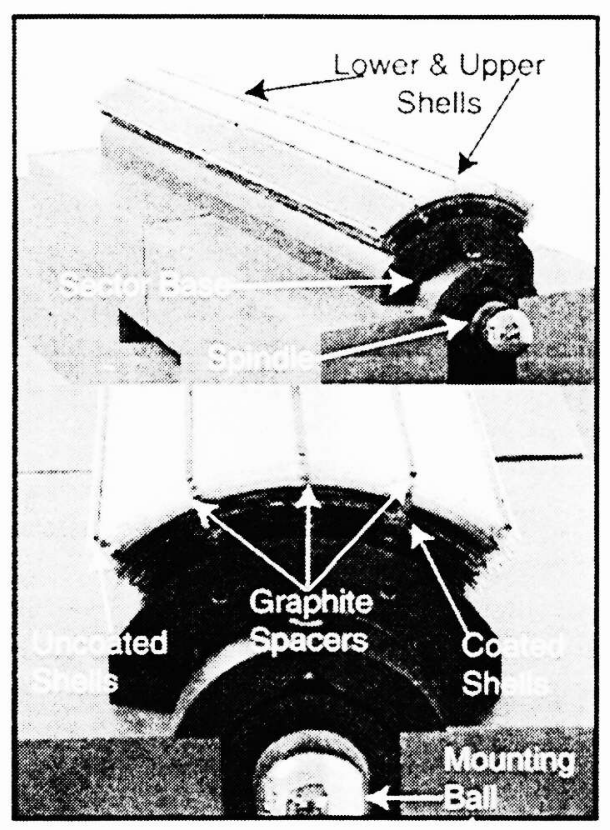

Figure 2. Photograph of the 2-bounce glass prototype being assembled. Both coated and uncoated optics are included for direct figure comparison.

to maintain good figure, thereby achieving the mass goal. The Harvard-Smithsonian Center for Astrophysics has developed a coating chamber for depositing multilayers directly on the interiors of cylinders, and has demonstrated that high-reflectance multilayers can be deposited on relatively large-radius $(r \gtrsim 12$ cm) optics (Figure 3 ). In an alternate approach, Northwestern University and U. Brera are independently developing a technicue for replication of the multilaver itself. In this technique, the multilayer is deposited directly on the mandrel, the nickel is then formed on top of the coating, and the entire optic released from the mandrel. To-date, this has successfully been demonstrated using flats. ${ }^{7}$
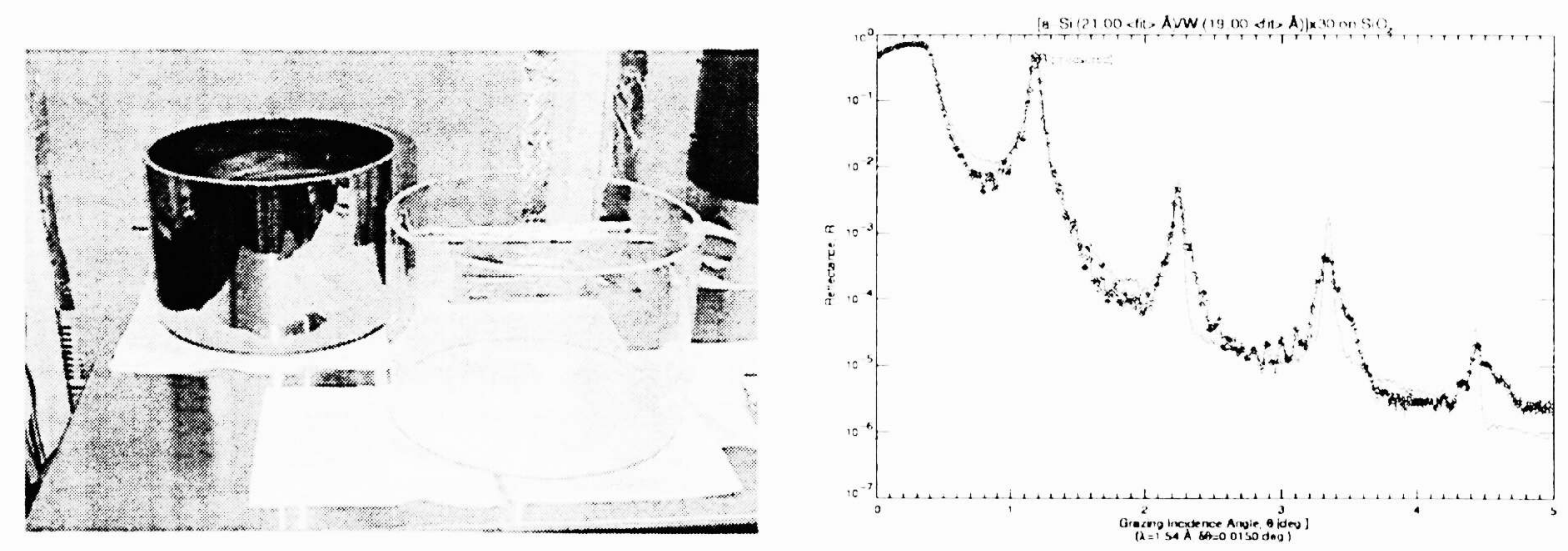

Figure 3. Photo shows coated and uncoated 10.5 inch diameter Duran glass substrates used for testing. Plot is $8 \mathrm{keV}$ reflectivity data (points) for the optic shown here along with fit to the data (solid line) using the following parameters: $\mathrm{N}=30$ bilayers $\mathrm{W} / \mathrm{Si}, \mathrm{d}=40.0$, gamma $=0.475, \sigma_{W / S}=3.5 \AA . A, \sigma_{S / W}=5.0 \AA$. 


\begin{tabular}{|l|l|}
\hline$\Delta \mathrm{X}$ & $\leq 730 \mu \mathrm{m}$ \\
\hline$\Delta E / \mathrm{E}$ & $<20 \% \mathrm{FWHM}(6-30 \mathrm{keV})$ \\
& $<10 \% \mathrm{FWHM}(\mathrm{E}>30 \mathrm{keV})$ \\
\hline Diameter & $\geq 2.3 \mathrm{~cm}$ \\
\hline Quantum Efficiency & $\geq 90 \%(6-40 \mathrm{keV})$ \\
\hline Internal Background & $<2 \times 10^{-4} \mathrm{cts} / \mathrm{cm}^{2} / \mathrm{s} / \mathrm{keV}$ \\
\hline
\end{tabular}

Table 2. Required detector specifications.

\subsection{Multilayers}

Depth-graded multilayers with sufficient reflectivity have been deposited, using magnetron sputtering, on both segmented mirror substrates and on curved pyrex cylinders. For Constellation-X, W/Si is a good material combination, in that there is high contrast in refractive index between the materials, the films can be grown with small interface widths (typically $3-3.5 \AA$ ), and good reflectance extends to the tungsten K-edge at $69.5 \mathrm{keV}$ (see Figure 4). Typical parameters for the Con-X W/Si designs are $300-500$ layer pairs, minimum period of $20 \AA$, and maximum period of $200 \AA$. However, if response significantly above the $40 \mathrm{keV}$ baseline requirement is desired, alternate material combinations, such as $\mathrm{Pt} / \mathrm{C}$ (cutoff at $78 \mathrm{keV}$ ) or $\mathrm{Ni} / \mathrm{C}$ must be used. Aside from the replication issues associated with applying the films to the interiors of small-radius optics, there are no remaining technical hurdles associated with the Con-X multilayers.

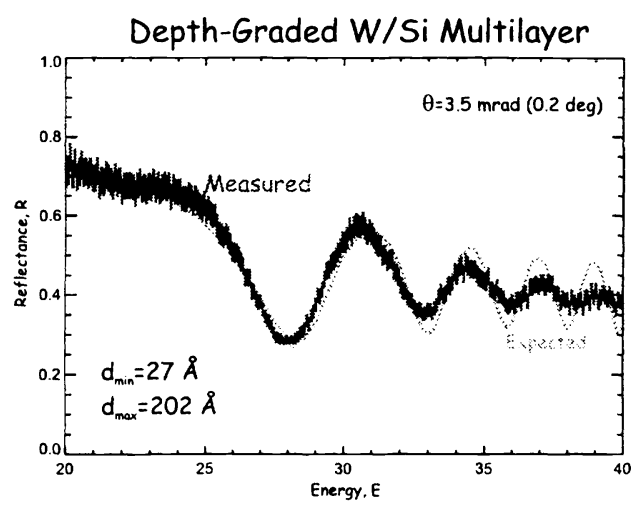

Figure 4. Measured reflectance of a depth-graded $W / S i$ multilayer deposited on a formed glass substrate vs. energy (solid line), compared to a theoretical calculation (dotted line). Typical interface widths achievable for W/SI multilayers are $\sigma_{i f}=3.0-3.5 \AA$.

\subsection{Detectors}

Table 2 summarizes the detector parameters required to meet the baseline performance specifications described in Section 2 for the $10 \mathrm{~m}$ telescope focal length. The pixel size of $730 \mu \mathrm{m}$ (or less) corresponds to $15^{\prime \prime}$, nicely oversampling the $1^{\prime}$ HPD mirror response. The background specification is set by the requirement that typical $2 \times 10^{4} \mathrm{~s}$ observations be signal-limited, assuming a $1^{\prime}$ HPD. The requirement for good quantum efficiency is particularly important at high energies, where the mirror response is decreasing rapidly.

The primary choice for the focal plane detectors is a high-atomic number solid state pixel detector such as $\mathrm{CdZnTe}$ or CdTe. The detector is hybridized: the anode contact is segmented into pixels, with each pixel bump bonded to a separate channel of an VLSI circuit (see Figure 5). The pitch of readout circuits on the chip matches that of the pixels on the solid state sensor. Stacked silicon strip detectors are also being studied as a backup technology. The motivation for considering these sensors is the superior resolution at low energy, and the good uniformity of the material. Achieving the requisite quantum efficiency at $40 \mathrm{keV}$ requires stacking 10 detectors.

The three principal technical issues associated with demonstrating CdZnTe sensors with the Con-X requirements are: 1) obtaining defect-free sensor material with the requisite dimensions, 2) developing a low-noise readout circuit 


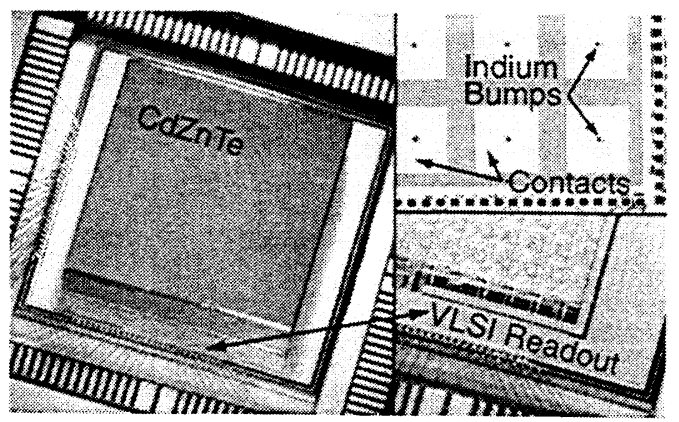

Figure 5. Photo of a prototype CdZnTe hybrid, showing detector geometry and packaging.

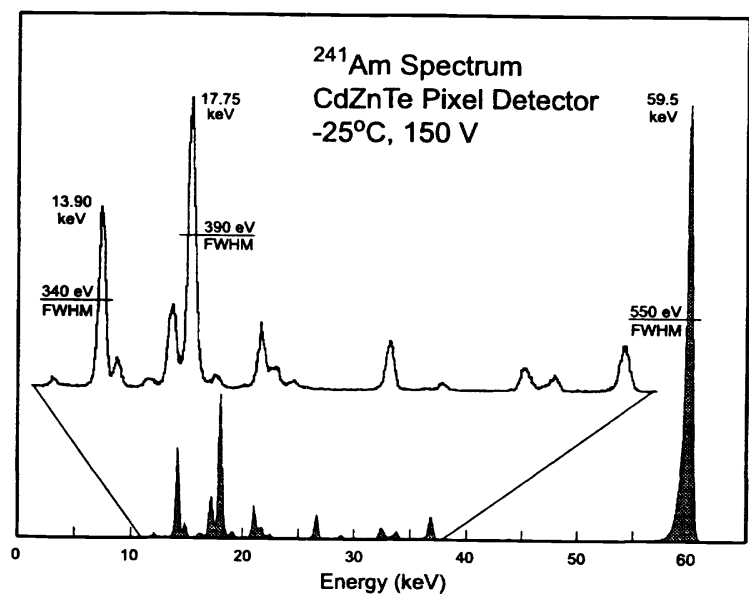

Figure 6. Spectrum of an ${ }^{241} \mathrm{Am}$ source taken with a $650 \mu \mathrm{m}$ CdZnTe pixel sensor coupled to a custom low-noise VLSI readout. The detector was at $-20 \mathrm{deg} \mathrm{C}$, similar to the operating temperature expected for Con-X.

capable of achieving a threshold below $6 \mathrm{keV}$, and 3) demonstrating good quantum efficiency for CdZnTe and CdTe sensors at low energy. Significant progress has been made recently on all three areas. An effort lead by GSFC has demonstrated that defect-free 1-inch CdZnTe sensors can be selected and diced from wafers. The wafers were procured from the manufacturer, eV Products, imaged in IR and scanned using an X-ray beam at GSFC to select the material. Although the yield is not high, given the relatively small number of detectors required by Con-X, this approach is feasible for selecting flight detectors. In addition, a custom low-noise VLSI readout coupled to a $650 \mu \mathrm{m}$ CdZnTe pixel sensor, developed for the CIT/Columbia/DSRI HEFT balloon program, has demonstrated a threshold below $2 \mathrm{keV}$. The quantum efficiency at these low energies has not yet been fully characterized.

\subsection{Background calculations}

The internal detector background is critical to understand, since it determines, for a fixed HPD, the integration time for which observations will be signal-limited. As part of the Constellation-X technology development program, we have studied the contributions to the background in CdZnTe sensors using a baseline shielding configuration for the baseline Lagrange-point orbit. The Monte Carlo calculations, performed by MSFC and SAIC Corp., included contributions from the Galactic cosmic rays as well as trapped protons, and all particles produced in secondary interactions in the shield and spacecraft.

We employed a simplified model of the detector and shield. The shield consisted of a $31 \mathrm{~cm} \mathrm{high,} 2.51 \mathrm{~cm}$ radius, $1.9 \mathrm{~cm}$ wall-thickness active $\mathrm{BGO}$ well, and we assumed a $0.1 \mathrm{~cm}$ thick, $1.4 \mathrm{~cm}$ radius detector positioned $4 \mathrm{~cm}$ from the bottom of the shield. It is important to emphasize that the shield geometry has not yet been optimized to minimize the background. The results of the Monte Carlo find that the levels of the most significant 


\begin{tabular}{|c|c|c|}
\hline \multicolumn{3}{|c|}{ Telescope } \\
\hline focal length & \multirow{3}{*}{\multicolumn{2}{|c|}{$\begin{array}{c}10 \mathrm{~m} \\
4 \\
3\end{array}$}} \\
\hline no. of satellites & & \\
\hline $\mathrm{hxt} /$ satellite & & \\
\hline \multicolumn{3}{|c|}{ Multilayers } \\
\hline materials & \multirow{3}{*}{\multicolumn{2}{|c|}{$\begin{array}{c}\text { W/Si } \\
20 \AA \\
500\end{array}$}} \\
\hline minimum period & & \\
\hline max. no. of layers & & \\
\hline \multicolumn{3}{|c|}{ Optics } \\
\hline & glass & nickel replica \\
\hline minimum radius & $3 \mathrm{~cm}$ & $6 \mathrm{~cm}$ \\
\hline maximum radius & $20 \mathrm{~cm}$ & $20 \mathrm{~cm}$ \\
\hline shell length & $25 \mathrm{~cm}$ & $40 \mathrm{~cm}$ \\
\hline shell thickness & $300 \mu \mathrm{m}$ & $100 \mu \mathrm{m}$ \\
\hline no. of shells/module & 149 & 82 \\
\hline mirror mass/satellite & 190 & 250 \\
\hline
\end{tabular}

Table 3. Optics parameters used to calculate performance for the example designs.

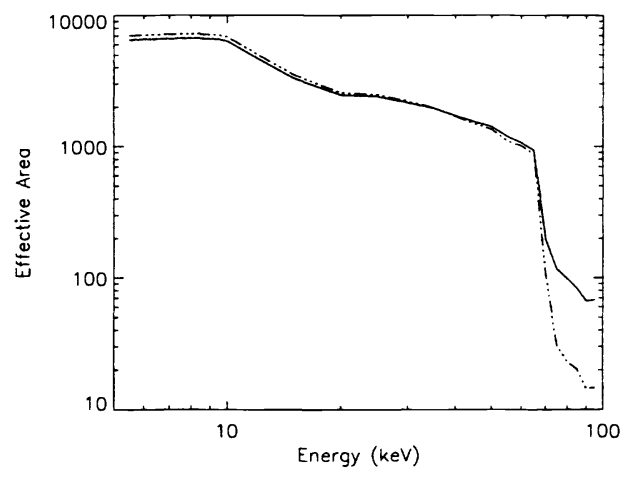

Figure 7. Effective area vs. energy for the segmented glass (solid line) and nickel replica (dot-dashed line) example configurations.

internal background components (excluding diffuse aperture flux, which must be collimated) are, in order: shield activation: $7 \times 10^{-5} \mathrm{cts} / \mathrm{cm}^{2} / \mathrm{s} / \mathrm{keV}, \mathrm{CdZnTe}$ activation: $7 \times 10^{-6} \mathrm{cts} / \mathrm{cm}^{2} / \mathrm{s} / \mathrm{keV}$, and prompt background due to cosmic ray interactions: $3 \times 10^{-6} \mathrm{cts} / \mathrm{cm}^{2} / \mathrm{s} / \mathrm{keV}$. With appropriate collimation of the diffuse flux, the total predicted background is at the $10^{-4} \mathrm{cts} / \mathrm{cm}^{2} / \mathrm{s} / \mathrm{keV}$ level, sufficiently low to meet the baseline requirement. It is encouraging that the dominant component is from shield activation, so that optimization of the shield thickness can likely reduce the total internal background further.

\section{EXAMPLE DESIGNS}

To demonstrate that the desired performance is achievable within the physical envelope using realistic extrapolations of existing technologies, we have derived example designs based on both segmented and integral mirror shells. Table 3 shows the technical parameters assumed for the glass segmented and nickel replica mirrors. In both cases we assume detector quantum efficiency of $90 \%$ independent of energy. The W/Si multilayer design was optimized for the Con-X energy range and FOV using the procedure described in Mao et al. (1999).

We have chosen the mirror parameters for glass and nickel substrates to reflect the different technical limitations inherent in each technology. The minimum radius for nickel is chosen to be large enough that multilayers can be directly deposited on the interior. The shell length for nickel is also longer in order to reduce the number of mandrels 
required for the replication (the longer shells reduce the total number of mirrors). The largest technical extrapolation is in the thickness of nickel, assumed to be $100 \mu \mathrm{m}$ independent of radius. Good angular resolution has not been demonstrated for shells this thin, however new nickel alloys developed for the SXT are promising for achieving this goal.

Figure 7 shows the effective area vs. energy for the two mirror configurations. Both achieve the requisite collecting area of $1500 \mathrm{~cm}^{2}$ at $40 \mathrm{keV}$. Some reduction in the nickel mass is still required in order to meet the mass restriction, however this may be possible by thinning the inner mirror shells further.

\section{CONCLUSION}

Significant progress has been made over the last few years on the development of the technologies required for the Con-X HXT. With the demonstration of high-reflectance depth-graded multilayers, appropriate X-ray optics, and position-sensitive high-atomic number solid state detectors, no serious technical hurdles remain. Several aspects must, however, still be demonstrated. These include: a glass prototype mirror demonstrating $1^{\prime}$ or better over the entire aperture, nickel mirrors of appropriate radius $\leq 100 \mu \mathrm{m}$ thick, and high quantum efficiency for CdZnTe pixel detectors at photon energies below $10 \mathrm{keV}$.

\section{REFERENCES}

1. P. J. Serlemitsos and Y. Soong, "Foil x-ray mirrors," Astrophys. and Sp. Sci. 239, pp. 177-196, 1996.

2. O. Citterio and P. L. Jensen, "High throughput replica x-ray optics," Proc. SPIE 1140, pp. 337-0, Oct. 1989.

3. A. M. Hussain, F. E. Christensen, M. A. Jimenez-Garate, W. W. Craig, T. A. Decker, C. J. Hailey, M. Stern, F. A. Harrison, G. Pareschi, A. Souvorov, M. S. D. Rio, R. Tucoulou, and A. K. Freund, "X-ray scatter measurements from thermally slumped thin glass substrates for the heft hard x-ray telescopes," Proc. SPIE 3766, 1999.

4. W. W. Craig, F. E. Christensen, T. A. Decker, C. J. Hailey, F. A. Harrison, R. M. Hill, M. A. Jimenez-Garate, P. H. Mao, and S. M. Schindler, "Hard x-ray optics for the heft balloon-borne payload: prototype design and status," Proc. SPIE 3445, pp. 112-120, Nov. 1998.

5. P. H. Mao, F. A. Harrison, Y. Y. Platonov, D. Broadway, B. Degroot, F. E. Christensen, W. W. Craig, and C. J. Hailey, "Development of grazing incidence multilayer mirrors for hard x-ray focusing telescopes," Proc. SPIE 3114, pp. 526-534, Oct. 1997.

6. L. V. Speybroeck, O. Citterio, S. L. O'Dell, R. Petre, and B. Ramsey, "Constellation-x spectroscopy x-ray telescope: requirements and development program," Proc. SPIE , 1999. these proceedings.

7. O. Citterio, G. Pareschi, E. Poretti, C. Misiano, and F. Pozzilli, "Multilayer optics for hard x-ray astronomy by means of replication techniques," Proc. SPIE 3766, 1999.

8. P. H. Mao, F. A. Harrison, D. L. Windt, and F. E. Christensen, "Optimization of graded multilayer designs for astronomical x-ray telescopes," Applied Optics 38, p. 4766, 1999. 